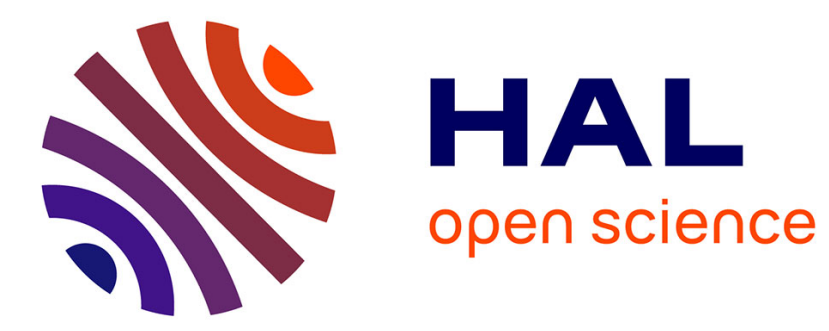

\title{
Virtual Age Models: Monitoring Information Level and Quality of Parametric Estimation
}

Léa Brenière, Christophe Bérenguer, Laurent Doyen

\section{To cite this version:}

Léa Brenière, Christophe Bérenguer, Laurent Doyen. Virtual Age Models: Monitoring Information Level and Quality of Parametric Estimation. RAMS 2021 - Annual Reliability and Maintenability Symposium, May 2021, Orlando, United States. pp.1-6, 10.1109/RAMS48097.2021.9605776 . hal03442946

\section{HAL Id: hal-03442946 \\ https://hal.science/hal-03442946}

Submitted on 23 Nov 2021

HAL is a multi-disciplinary open access archive for the deposit and dissemination of scientific research documents, whether they are published or not. The documents may come from teaching and research institutions in France or abroad, or from public or private research centers.
L'archive ouverte pluridisciplinaire HAL, est destinée au dépôt et à la diffusion de documents scientifiques de niveau recherche, publiés ou non, émanant des établissements d'enseignement et de recherche français ou étrangers, des laboratoires publics ou privés.

\section{(ㅇ)(1) $\$$}

Distributed under a Creative Commons Attribution - NonCommercial - NoDerivatives $\mid 4.0$ 


\title{
Virtual Age Models: Monitoring Information Level and Quality of Parametric Estimation
}

\author{
Léa Brenière, Univ. Grenoble Alpes, CNRS, Grenoble INP, LJK, 38000 Grenoble, France \\ Christophe Bérenguer, PhD, Univ. Grenoble Alpes, CNRS, Grenoble INP, GIPSA-Lab \\ Laurent Doyen, PhD, Univ. Grenoble Alpes, CNRS, Grenoble INP, LJK, 38000 Grenoble, France
}

Key Words: inspections frequency, parametric estimation, time-dependent covariates, virtual age models

\section{SUMMARY \& CONCLUSIONS}

An industrial system is subject to failures during its lifetime. These failures are corrected by maintenances, and then the system works again until the next failure. However, the system is not necessarily in the same state as just before the failure. A typical assumption is the As Bad As Old hypothesis, where the system stays in the same state after the failure. Another hypothesis is the As Good As New case, in which the system is totally renewed after a failure. But between minimal and perfect repairs, there exists a wide range of imperfect maintenances. Virtual age models are a way to deal with an imperfect maintenance effect. They also take into account the intrinsic wear of the system. Numeric estimation is used to infer the values of the parameters for some data set.

In some cases, we are able to observe several identical and independent systems. Then, we can record some differences between these systems. This information can be added in the virtual age model as covariates. However, we may lack the continuous observation of the covariates. For example, periodic inspections of the systems will only provide discrete covariates, that we can use in the estimation as step-wise constant covariates. The first purpose of this paper is to understand how the frequency of inspection of the covariates impacts the quality of the parametric estimation.

Too frequent inspections of the systems could lead to unexpected and unwanted costs. The frequency of inspection should be balanced so that their cost is reasonable compared to the risk of a bad quality estimation. The finding of an optimal cost is the second aim of this paper.

\section{INTRODUCTION}

An industrial system can face failures and maintenances throughout its lifetime. After a failure, a repair is immediately carried out and the system is switched on after a negligible down time. This is called corrective maintenance. One can also think of a patient facing relapses of their disease, for example a carcinoma or migraines, with medical treatments viewed as maintenances. This type of data is called recurrent events data and specific methods apply. In our work, we do not take into account the structure of the system nor the role of single components: the whole system is viewed as a unique entity. In such situations, the conditional failure intensity is a very useful mathematical tool which characterizes the distribution of the failure process. One basic assumption is the Non Homogeneous Poisson Process, where the conditional intensity is a deterministic function of time. In this case, the system after a repair is As Bad As Old (ABAO), which means the maintenance did not put the system in a better state than just before the failure. This maintenance is said to be minimal. Another basic assumption is the Renewal Process, where the interfailure times are independent and identically distributed. In that case, the system after a repair is As Good As New (AGAN), which means the maintenance was able to totally renovate the system. But these two assumptions may be too strong. In between, there is what is called imperfect maintenance. For example, when a single component is replaced, the system may be better than old but worse than new. The effect of the maintenance on the whole system is therefore imperfect. Generalized virtual age models [1] can be used to take into account both the intrinsic wear of the systems and the effect of the maintenances. The former is specified with a parametric initial intensity, which corresponds to the hazard rate of the first failure time. The latter is specified with a virtual age that depends on a maintenance efficiency parameter. The virtual age, or effective age, is to be viewed as different from the calendar age of the system, which is merely the time elapsed since it was turned on. Different forms of virtual age can be considered. With a minimal repair, the virtual age is equal to the calendar age. With a perfect repair, the virtual age is equal to the time elapsed since the last repair. The general idea for imperfect maintenance is that after a repair, the system is in a state equivalent to a new one that survived until the virtual age of the studied system [2].

When several identically distributed and independent systems are considered at the same time, it is of interest to record information that may differ between the systems and influence their failure process. For an industrial system, we can think of the manufacturer, the work load, the speed of the engine, the weather conditions etc. For a patient, the gender, the body temperature or the size of the tumor could also carry useful information about the failure process. This is known as observed heterogeneity between the systems. All this 
information can be integrated into generalized virtual age models as covariates [3]. We may have constant covariates (such as the system manufacturer or the patient gender) or timedependent ones (like the weather conditions or the body temperature). A simulation method and an estimation procedure in this framework have been developed in [3], in the case of step-wise constant covariates. To the best of our knowledge, very few references include unobserved heterogeneity in virtual age models (see [4] and [5]). In this work, we will focus on the use of covariates.

Continuous covariates can be incorporated in the model as step-wise constant ones with a short time step. However, the whole covariates may not be recorded. For example, with continuous covariates, we may be able to inspect the systems only at given discrete times to record the current values of the covariates. We may then face a certain lack of information, that has an impact on the quality of estimation. The aim here is to understand the behavior of the estimation with respect to the frequency of inspection of the covariates. It is expected that the more information we have, that is, the more often we inspect the system, the better the estimation is. This reasonable guess has to be statistically studied. This is one of the purposes of this paper.

Even though the more information we have, the higher the quality of estimation is, we may not want to inspect the systems too often. Indeed, the inspections may have a cost that has to be taken into account when planning them. Therefore, the maintainer should balance the cost of a high frequency inspection planning and the cost of an erroneous estimation. An optimum can be found here: a point where more inspections would cost more than the gained quality of estimation and where less inspections would lead to a too much degraded estimation. We present in this paper an optimization of the inspection frequency so that maintainers are able to survey covariates in an optimal way and to get worthwhile estimations.

In this paper, we recall the mathematical framework allowing the simulation of new data sets and the estimation of the model parameters in a first part. Then we study the quality of estimation with respect to the frequency of inspection of a time-dependent covariate. Finally, we set up a method to find the optimal frequency of inspection of the systems. Conclusions will end the article.

\section{MATHEMATICAL FRAMEWORK}

\subsection{Conditional failure intensity and virtual age}

The core concept of the mathematics involved in our work is the conditional failure intensity. It is a characterization of the distribution of the next failure time, given the past of the process. Let us assume that we observe the $n$ first failure times of a single repairable system $T_{1}, T_{2}, \ldots, T_{n}$, with $T_{0}=0$. We denote $\left(N_{t}\right)_{t \geq 0}$ the counting process of failures. Then the conditional failure intensity is given by:

$$
\lambda_{t}=\lim _{\Delta t \rightarrow 0} \frac{1}{\Delta t} P\left(N_{t+\Delta t}-N_{t^{-}}=1 \mid \mathcal{H}_{t^{-}}\right),
$$

where $\mathcal{H}_{t^{-}}$is the past of the process up to time $t$.

We will work here with a particular case of counting process called virtual age models. The idea of these models has been introduced in [1]: after a failure, the system is in the same state as if it had never failed and as if it had survived until a time called the virtual age. We denote this quantity $V_{i}(t)$, where $t$ is the calendar time and $i$ is the number of failures faced by the system. Here we will focus on models expressed in the following way:

$$
\lambda_{t}=V_{N_{t^{-}}}^{\prime}(t) h\left(V_{N_{t^{-}}}(t)\right),
$$

where $h(\cdot)$ is the failure rate of a new unmaintained system. This generic model was exposed in [2]. The virtual age $V_{i}(t)$ depends on a maintenance efficiency parameter $\rho$. The initial failure rate, $h(\cdot)$, can also depend on some parameters. Here, we will use a Power Law Process (PLP) with intensity

$$
h(t)=\frac{\beta}{\eta}\left(\frac{t}{\eta}\right)^{\beta-1},
$$

where $\eta>0$ is a scale parameter and $\beta>1$ is a shape parameter.

\subsection{Some virtual age models}

We work here with three particular cases of virtual age models. The first one is the $A R A_{\infty}$ model (Arithmetic Reduction of Age with infinite memory), obtained from (2) with the virtual age expression given by:

$$
V_{i}(t)=t-\rho \sum_{k=0}^{i-1}(1-\rho)^{k} T_{i-k},
$$

for all $0 \leq i \leq n$ and $t>T_{i}$, with the convention that the sum is null if $i=0$. The second model is the $A R A_{1}$ model (with memory one), obtained from (2) with the virtual age expression given by:

$$
V_{i}(t)=t-\rho T_{i},
$$

for all $0 \leq i \leq n$ and $t>T_{i}$. In the two $A R A$ models, the parameter $\rho$ corresponds to the efficiency of the maintenance. If $\rho=1$, the repair is perfect and the system is AGAN. If $\rho=$ 0 , the repair is minimal and the system is ABAO. If $\rho<0$, the repair is harmful, but we will not consider this case in our paper, so that $\rho \in[0,1]$. Therefore, the maintenance is somewhere between minimal and perfect. The last model we will use is the $Q R$ model, proposed in [6]. In this model, the interfailure times decrease or increase geometrically. It is obtained from (2) with the virtual age expression given by:

$$
V_{i}(t)=\frac{t-T_{i}}{\rho^{i}},
$$

for all $0 \leq i \leq n$ and $t>T_{i}$. The parameter $\rho$ corresponds again to the efficiency of the maintenance. If $\rho=1$, the repair is perfect and the system is AGAN. If $\rho \in] 0,1[$, the system deteriorates; if $\rho>1$, it improves.

\subsection{Covariates}

When we observe heterogeneity between several identical and independent systems, we can make use of covariates to deal with this information in the virtual age model. These covariates are associated to a coefficient which measures the influence of each covariate on the failure process. The covariates and the coefficient are included in the virtual age model with an exponential function, as described in [7]. The resulting intensity is the following:

$$
\lambda_{t}=V_{N_{t^{-}}}^{\prime}(t) h\left(V_{N_{t^{-}}}(t)\right) \exp \left(\gamma^{\prime} X_{t}\right),
$$


where $X_{t}$ are the time-dependent covariates and $\gamma$ is the coefficient of the covariates.

\subsection{Simulation}

In order to simulate data sets of failures times following the given model, we perform an inversion of the cumulative distribution function of the failure times. Since these failures times are not independent, we need to work with the conditional version of this function. In [3], a whole procedure for the simulation of new failure times was given. The main issue was that, with a random covariate, the inversion of the cumulative distribution function is not analytically possible. The given solution was to discretize the covariate so that it was step-wise constant. With the following mapping expression:

$$
X_{t}=\sum_{l=1}^{L} \chi_{l} 1_{\left[\tau_{l-1}, \tau_{l}[\right.}(t)
$$

we obtain an explicit expression of the conditional cumulative distribution function and of its generalized inverse, which we can use to simulate new failure times. Another simulation method was given in [3], which relies on an approximation of the probability of failure on small time intervals. Here we will use the first method.

\subsection{Estimation}

When we are given a data set, we need to know the values of the model parameters. To do so, we perform a parametric estimation, that is done here by maximizing numerically the log-likelihood, with the same assumption as in 2.4: the covariate is step-wise constant. The exact computation of the log-likelihood is given in [3].

\subsection{Example of a simulated dataset}

We simulate a dataset containing 3 independent systems. First, we simulate three covariate paths with different parameters. On Figure 1, we can clearly see the difference

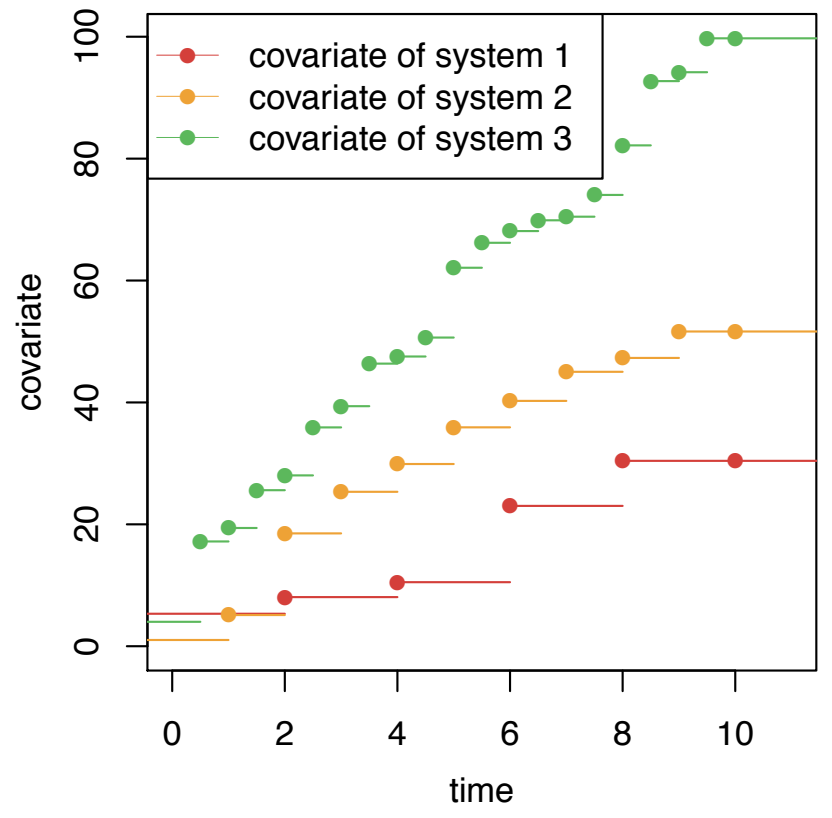

Figure 1-Three simulated covariate paths

between the simulated paths: the covariates have higher or lower increase rates. Then we simulate three realizations until time $t=10$ of the failure process, each being associated to one of the covariate paths. On Figure 2, we have represented the counting process of the three systems. We immediately feel the influence of the covariate when looking at the counting processes: for the greater covariate, there are 175 failures at time $t=10$, whereas for the lower one, there are only 4 failures, and 17 failures for the system in between.

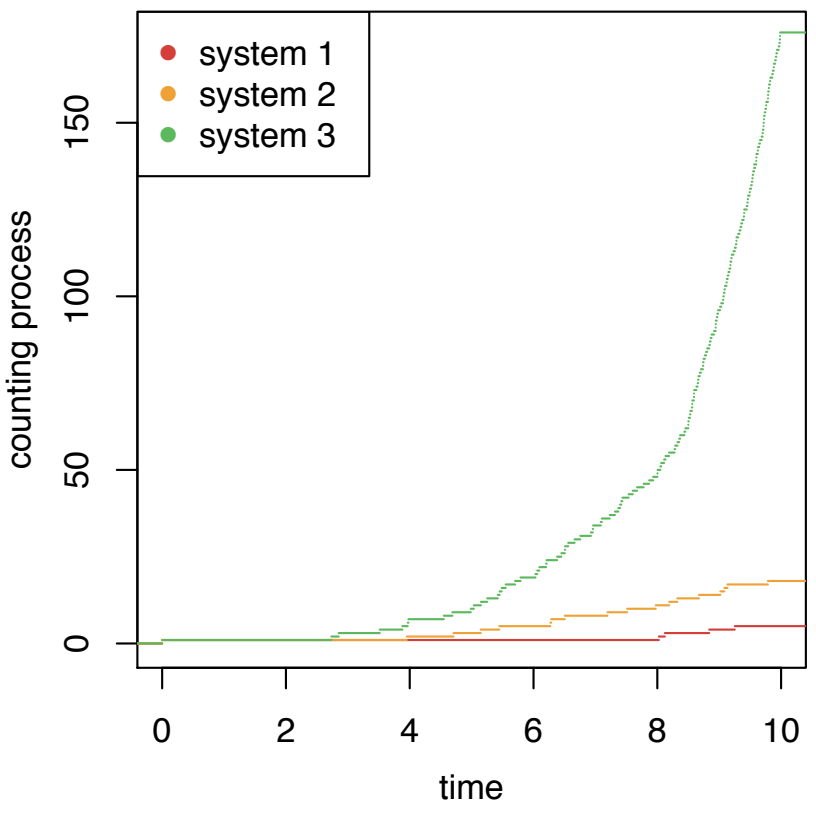

Figure 2 - Counting processes of three systems

Now we can try to estimate the model parameters, which are the failure intensity parameters, that is the shape and scale of the PLP, the efficiency of maintenances and the coefficient of covariates. We first use the whole covariate paths (Est 1), then we truncate the covariate so that we observe it each unit of time (Est 2), and finally each 2 units of time (Est 3). Intuitively, covariate 1 will be completely known, whatever the case is, since it jumps every 2 units of time. On the contrary, we will miss information on covariate 3 in case Est 2 and Est 3, since it jumps every 0.5 units of time. This lack of information will disturb the estimation.

With the estimation procedure explained in Section 2.5 and detailed in [3], we get three sets of estimations, that have to be compared to the true parameters used for the simulation. We give all these values in Table 1 .

Table 1 -Estimations of the parameters

\begin{tabular}{|c|c|c|c|c|}
\hline & $\eta$ & $\beta$ & $\rho$ & $\gamma$ \\
\hline True & 21.54 & 3 & 0.35 & 0.2 \\
\hline Est 1 & 24.04 & 3.33 & 0.31 & 0.22 \\
\hline Est 2 & 10.58 & 1.64 & 0.57 & 0.10 \\
\hline Est 3 & 9.18 & 1.24 & 0.78 & 0.08 \\
\hline
\end{tabular}

The estimations are close to the true values when using the whole covariate, whereas they get poorer when we use truncated paths. This difference in quality with respect to the inspection frequency is the subject of the next section. 


\section{QUALITY OF ESTIMATION}

\subsection{Goal of the study}

A thorough study of the quality of the parametric estimation was provided in [3]. However, it was done with a completely known covariate. In the case where we miss some information about the covariate, this study is no longer relevant. For example, the monitoring of the working conditions of the systems may give only values at discrete times. We can assume that the covariates are constant in between, but then we lack some information and it is expected that the estimation will not be as good as in the case of a completely known covariate. We choose here to work with periodic inspections of the systems conditions. Then, we want to study the impact of the frequency of estimation on the quality of estimation. The estimation procedure is not limited to a single covariate. However, for the sake of simplicity, we chose to work here with only one covariate.

To do so, we focus on some estimation error statistics. The first one is the Mean Squared Error (MSE), the others are based on the simulation of the next failure time. We simulate the next failure time with estimated parameters either with the whole covariate or a truncated covariate. The two samples of next failure times are compared with the two-sample KolmogorovSmirnov statistic [8] or with the respective sample means. This is a way to measure the distance between the law given by the estimations with a whole covariate or a truncated one. The lower these estimation error statistics are, the better the quality of estimation is.

\subsection{Computation of the estimation error statistics}

Here is how we compute the five estimation error statistics.

- We simulate a covariate and a data set with fixed parameters.

- We estimate the parameters of the model with the complete covariate.

- For each system $j$, we simulate a sample of the next failure time with these parameters and we take the mean $m_{j}$ of this sample.

- We truncate the covariate with respect to the inspection interval.

- We estimate the parameters of the model with the truncated covariate.

- For each system $j$, we simulate a sample of the next failure time with these parameters and we take the mean of this sample $m_{j}^{t r}$ We also compute the Kolmogorov-Smirnov statistics $K S_{j}$ of discrepancy between this sample and the one we got with the complete covariate.

- We compute $K S^{\text {mean }}, K S^{\text {med }}, K S^{\text {max }}$, respectively the mean, the median, and the maximum of the $K S_{j}$ statistics over all the systems, and the mean $M_{\text {diff }}$ over all the systems of diff $f_{j}=\left|m_{j}-m_{j}^{\text {tr }}\right|$.

- We simulate and repeat this whole procedure $M=1000$ times.

- We take the mean over all the simulated data sets of $K S^{\text {mean }}, K S^{\text {med }}, K S^{\max }, M_{\text {diff }}$, and we also compute the sum of the MSE of the four parameters $(\eta, \beta, \rho, \gamma)$.
These four means and the sum of MSE are our five estimation error statistics, which measure in a sense the inverse of quality of the parametric estimation.

\subsection{Setup of the study}

In our study, we choose inspection intervals among $\{0.2,0.25,0.3,0.4,0.5,0.6,0.75,0.8,1,1.2,1.5,1.6,2,2.4,3\}$. We use the three models exposed in 2.2: $A R A_{\infty}, A R A_{1}$ and $Q R$ with a PLP initial intensity. Each simulated data set contains 50 systems facing 20 failures each, between times 0 and 24 . The data sets are therefore rather large, which may not be realistic. However, the goal here is to study the effect of inspections while being free of quality limitations due to data size. The unique covariate is simulated as a jump process with finite states space. The random jumps occur at intervals of mean 1 . The state space is defined by $\{-2,-1,0,1,2\}$. The model parameters are given in Table 2 . The sample of the next failure time for each system contains 1000 replications. All calculations were performed with $\mathrm{R}$ software, in combination with the Rcpp package.

Table 2-Model Parameters for the Simulation Study

\begin{tabular}{|c|c|}
\hline$A R A$ models & $Q R$ model \\
\hline$\gamma_{0}=1$ & $\gamma_{0}=1$ \\
\hline$\eta_{0}=1 /\left(0.025^{1 / 2.5}\right)$ & $\eta_{0}=1 /\left(0.087^{1 / 2.5}\right)$ \\
\hline$\beta_{0}=2.5$ & $\beta_{0}=2.5$ \\
\hline$\rho_{0}=0.7$ & $\rho_{0}=0.9$ \\
\hline
\end{tabular}

\subsection{Results}

In Figure 3, the plots of the different estimation error statistics versus the time between two inspections are displayed. For example, at the vertical line of abscissa 2, we can read the values of the statistics for the three studied models when there are 2 units of time between two inspections. We can observe what we expected: the five estimation error statistics are increasing with the inspection interval, with all studied models, at higher or lower rates, which means that the more frequent the inspections are, the better the quality of estimation is.

\section{OPTIMAL FREQUENCY OF INSPECTION}

\subsection{Goal and setup of the study}

Now we add a new dimension in our study: the cost. Indeed, frequent inspections can have a high cost, and the gain in terms of quality of estimation may not be worthwhile. Therefore, we compute a cost that balances the estimation error (measured with the five previous statistics) and the cost of inspections. We stay in the same design as in Section 3 and we build a cost function as the number of inspections (each inspection having value 1) plus the chosen statistic multiplied by a positive cost coefficient. This coefficient aims to make the two quantities commensurable. Here is the formula:

$$
C=C_{I} N_{I}+C_{E} S_{E},
$$

where $C$ is the total cost, $C_{I}$ is the cost of an inspection, $N_{I}$ is the number of inspections, $C_{E}$ is the cost of the estimation error and $S_{E}$ is the estimation error statistic. $C_{E}$ does not represent a physical cost. Its purpose is to make the two terms 

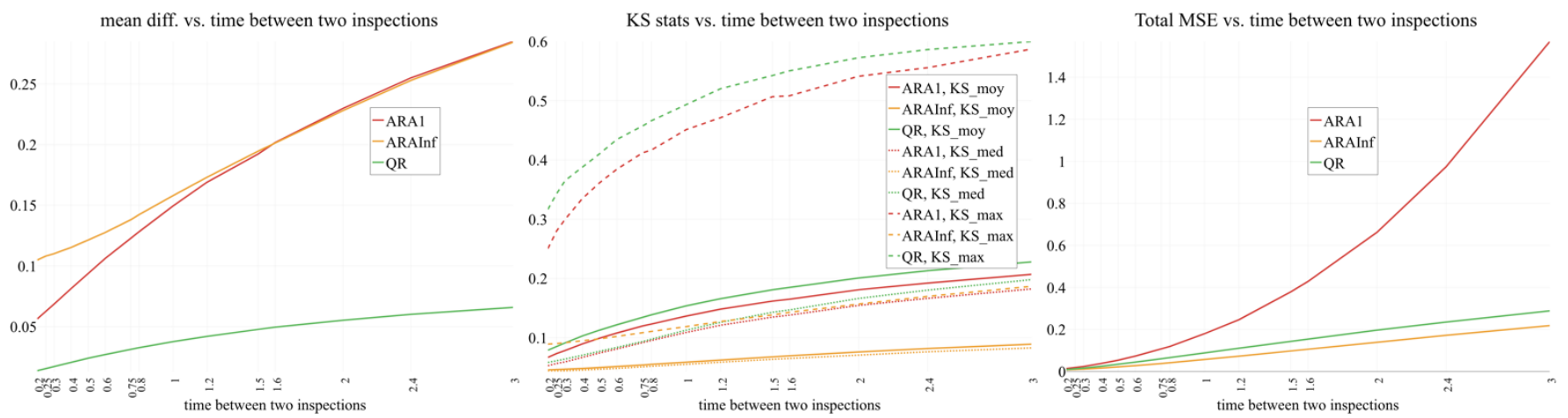

Figure 3 -Quality indicators with respect to the time between two inspections

commensurable and to include in the total cost the consequences of an estimation error, for example a bad decision. Then, the higher this statistic is, the higher the cost is. The number of inspections is trivially decreasing with respect to the inspection interval. We saw that our statistics are increasing with respect to the inspection interval. By balancing these two quantities, we should find the optimal inspection frequency in order to inspect not too often without degrading the quality of estimation. Therefore, we look for a minimum of our cost function.

\subsection{Results}

In Figure 4, an example is given with estimation error cost $C_{E}=500$ (which with our values of parameters, is able to make the quantities comparable, as stated in Section 4.1), for the five estimation error statistics and the three considered models. We observe in all cases (that is, with the five statistics and the three models) a minimum in the curves, which represents a trade-off between the cost of inspections and the cost of estimation error. The abscissa of this minimum is the inspection interval for which the minimum is obtained. This inspection interval is given in the legend of the plots.

Obviously, the weighting done by the estimation error cost is very important. In one extreme, if this cost is null, the total cost is equal to the number of inspections. Therefore, the minimum exists for the longest interval. It is easily understandable: if we do not account for the estimation error, we can proceed to as few inspections as possible. In the other extreme, for a sufficiently high cost, the number of inspections does not weigh anymore against the contribution of the estimation error in the cost. The optimization will then choose the lower error possible, that is for the smallest interval. Again, we can understand that since if the inspection does not cost much against the error of estimation, it is better to inspect very often so that we get a good quality estimation.

\section{CONCLUSIONS}

In this article, we studied the influence of the frequency of inspection on the quality of the parametric estimation, given a virtual age model with time-dependent covariates. First, we saw that the more frequent the inspections are, the better the quality of estimation is, in terms of five different statistics measuring the estimation error. Then, we saw that it is possible to find numerically an optimum in a cost function that balances the cost of inspections and the cost of the estimation error. With a wellchosen cost coefficient, we were able to find the inspection interval that minimizes the total cost.

This study is a first step toward maintenance planning. Indeed, we can think of a situation where we want to use the covariates in order to plan preventive maintenances. The first step in the procedure would be to estimate the parameters of the model, given a dataset. But then we need to know whether this estimation is of good quality, in particular if we can only inspect the systems at given discrete times. In [9], the virtual age models were used to optimize preventive maintenances. However, this article did not include covariates. An extension both of that work and of ours could be to incorporate dynamic covariates into the optimization procedure of [9].
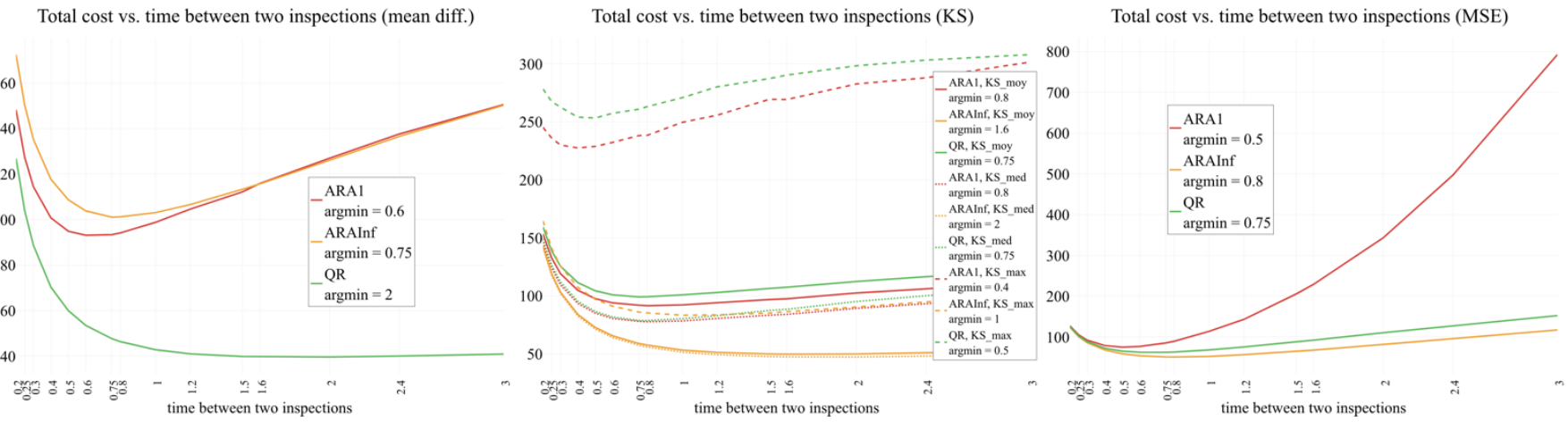

Figure 4-Total cost with respect to the time between two inspections 
1. M. Kijima, "Some results for repairable systems with general repair", J. Appl. probab. 26 (1) (1989) 89-102

2. L. Doyen, R. Drouilhet and L. Brenière, "A Generic Framework for Generalized Virtual Age Models", IEEE Trans. Reliab. 69 (2) (2020) 816-832

3. L. Brenière, L. Doyen and C. Bérenguer, "Virtual Age Models With Time-Dependent Covariates: A Framework for Simulation, Parametric Inference and Quality of Estimation”, Reliab. Eng. Syst. Saf. 203 (2020) 107054

4. P. Liu, E. A. Pena, "Dynamic Modeling \& Analysis of Recurrent Competing Risks and a Terminal Event", Stat. Stoch. Data Anal. Methods Appl. (2015) 111

5. X. Liu, J. Vatn, Y. Dijoux, H. Toftaker, "Unobserved heterogeneity in stable imperfect repair models", Reliab. Eng. Syst. Saf. 203 (2020) 107039

6. H. Wang, H. Pham, "A quasi renewal process and its applications in imperfect maintenance", Int. j. syst. sci. 27 (10) (1996) 1055-1062.

7. D. R. Cox, "Regression models and life-tables", J. Royal Stat. Soc.: Ser. B (Methodol.) 34 (2) (1972) 187-202

8. J. W. Pratt, J. D. Gibbons "Kolmogorov-Smirnov TwoSample Tests", In: Concepts of Nonparametric Theory. Springer Series in Statistics (1981) Springer, New York, NY.

9. G. L. Gilardoni, M. L. G. de Toledo, M. A. Freitas and E. A. Colosimo, "Dynamics of an optimal maintenance policy for imperfect repair models", Eur. J. Oper. Res. 248(3) (2016) 1104-1112.

\section{ACKNOWLEDGEMENTS}

This work has been partially supported by the LabEx PERSYVAL-Lab (ANR-11-LABX-0025-01) funded by the French program Investissement d'avenir.
Léa Brenière, PhD student

Laboratoire Jean Kuntzmann

Univ. Grenoble Alpes, CNRS, Grenoble INP, LJK

700 Avenue Centrale

38401 Saint-Martin-d'Hères, FRANCE

e-mail: lea.breniere@univ-grenoble-alpes.fr

Léa Brenière is a third-year Ph.D. student in applied mathematics at Laboratoire Jean Kuntzmann (Univ. Grenoble Alpes, CNRS Grenoble, France). Her thesis deals with probability and statistics applied to imperfect maintenance modeling.

Christophe Bérenguer, $\mathrm{PhD}$

GIPSA-lab

Univ. Grenoble Alpes, CNRS, Grenoble INP

11 Rue des Mathématiques

38402 Saint-Martin-d'Hères, FRANCE

e-mail: christophe.berenguer@grenoble-inp.fr

Christophe Bérenguer is a professor of reliability and maintenance engineering, systems monitoring and control systems at GIPSA-Lab (Univ. Grenoble Alpes, CNRS, Grenoble IINP - Grenoble, France).

Laurent Doyen, $\mathrm{PhD}$

Laboratoire Jean Kuntzmann

Univ. Grenoble Alpes, CNRS, Grenoble INP, LJK

700 Avenue Centrale

38401 Saint-Martin-d'Hères, FRANCE

e-mail: laurent.doyen@univ-grenoble-alpes.fr

Laurent Doyen is an Associate Professor at Univ. Grenoble Alpes, France. His research interests include probability and statistics applied to reliability, ageing, imperfect maintenance modeling, competing risks, and random processes in reliability. 